\title{
Tratamiento endovascular de injuria arterial iatrogénica
}

\author{
Endovascular treatment of iatrogenic arterial injury
}

\author{
Marcelo A. Agüero', Pablo D Liva', Gastón Pozzi', Jorge A. Baccaro', Matias J. Arévalo'
}

\section{RESUMEN}

La injuria arterial de los vasos del cuello es una complicación rara y grave de distintos procedimientos frecuentemente realizados en la práctica médica actual. Presentamos un caso de manejo endovascular de un paciente con perforación carotídea, por colocación inadvertida de introductor en sistema arterial del cuello.

Palabras claves: stent recubierto, carótida, injuria vascular.

\begin{abstract}
Neck vascular injury is a rare but severe complication of frequently performed procedures in current medical practice. We present a case of endovascular management of a patient with carotid perforation due to unrecognized arterial sheath placement.
\end{abstract}

Key words: stent-graft, endovascular, vascular injury.

Revista Argentina de Cardioangiología Intervencionista 2017;8(3):142-143

\section{CASO CLÍNICO}

Paciente de 69 años, hipertenso, con diagnóstico estenosis aórtica severa (gradiente medio $99 \mathrm{mmHg}$, área 0,6 $\mathrm{cm}^{2}$ ) y dilatación de raíz aórtica (48 $\mathrm{mm}$ de diámetro máximo en TC de tórax). En mayo de 2016 se realiza cirugía de reemplazo valvular aórtico con prótesis biológica más reemplazo de aorta ascendente con tubo recto. A las 24 hs de la cirugía se inicia anticoagulación. A las 48 hs del posoperatorio se retira vía venosa central yugular interna derecha presentando sangrado a nivel del sitio de punción, que no cede con compresión manual. Se realiza TC de cuello que evidencia hematoma en región lateral derecha del cuello con extensión a hueco supraclavicular de $11 \times 5 \mathrm{~cm}$. Evoluciona rápidamente con disnea progresiva y disfagia, sobreviniendo el paro respiratorio. Se fracasa al intentar intubación orotraqueal debido a la compresión y desvío de la vía aérea secundaria al hematoma. Se realiza cricotiroidectomía de urgencia y con maniobras de reanimación cardiopulmonar se consigue estabilizar al paciente.

Se realiza arteriografía de urgencia. Para ello se punza arteria femoral común derecha y se coloca introductor valvulado 5F. Se constata perforación de arteria carótida

\footnotetext{
1. Instituto de Cardiología de Corrientes "Juana F. Cabral".

$\triangle$ Correspondencia: Dr. Marcelo Agüero. Bolívar 1334. Tel/Fax 0379-4410000. Email:marceloaguer@gmail.com
}

primitiva derecha con extravasación activa de material de contraste al momento del estudio. La inyección de contraste a nivel de arteria carótida opacifica además el sistema venoso yugular del cuello (Figura 1). Esto indica como mecanismo de la injuria arterial a la transfixión de la arteria carótida antes de alcanzar la vena yugular.

Se lleva cuerda Amplatz de $260 \mathrm{~cm}$ hasta el tercio distal de arteria carótida primitiva derecha. Se intercambia introductor $5 \mathrm{~F}$ por vaina Terumo $7 \mathrm{~F}$ de $90 \mathrm{~cm}$ la cual se lleva hasta el origen de carótida primitiva derecha. Se administran 5000 UI de heparina Na. A través de la vaina se lleva stent graft ADVANTA 9-59 mm (Figura 2) y se lo impacta a $10 \mathrm{~atm}$. Se constata adecuado resultado angio-

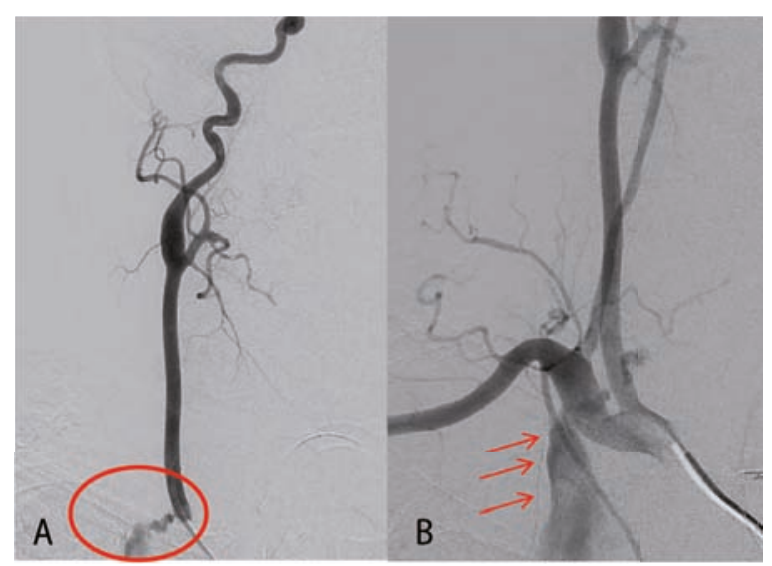

Figura 1. Angiografía basal: a) Extravasación activa de material de contraste en el tercio proximal de arteria Carótida Primitiva derecha. b) Opacificación de Vena Innominada derecha (felchas) a partir de la inyección de contraste en sistema arterial. 


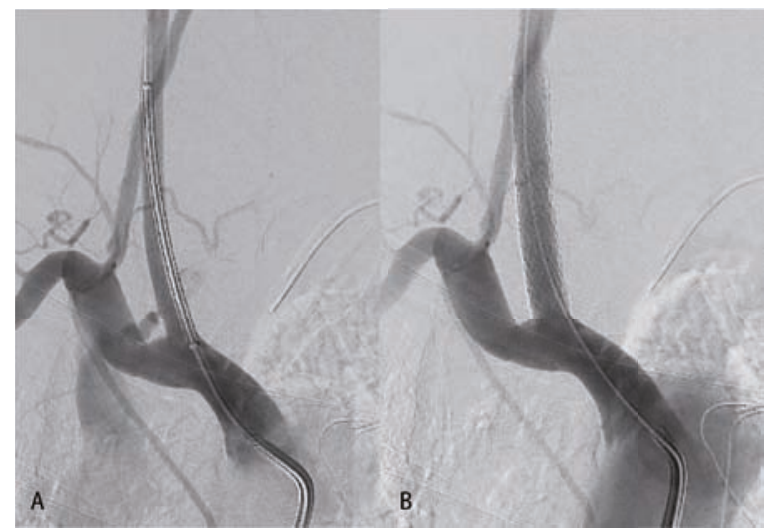

Figura 2. a) Posicionamiento de stentgraftAdvanta V12 9-59 mm en origen de Carótida Primitiva derecha. b) Resultado final: cese de la extravasación.

gráfico, con cese de la extravasación de contraste (Figura 2). Se administra protamina y se retira la vaina.

El 18/5/16 se logra decanulación traqueal, no presenta daño neurológico. Se reinicia anticoagulación vía oral. El 27/05/16 se realiza eco-Doppler de vasos de cuello de control que evidencia stent permeable y hematoma en vías de resolución. Evoluciona favorablemente decidiéndose su alta institucional al cumplirse el día 19 del posoperatorio.

\section{DISCUSIÓN}

Las injurias vasculares iatrogénicas pueden complicar diferentes procedimientos invasivos diagnósticos o terapéuticos. Su ocurrencia se ha incrementado de la mano de la expansión de dichos procedimientos en la práctica diaria, siendo responsable según reportes de hasta un tercio de todos los traumas vasculares ${ }^{1}$.

La canulación de una vena central se ha convertido en una práctica rutinaria en diferentes especialidades clínicas y quirúrgicas. De hecho, solamente en Estados Unidos se realizan cerca de 7 millones de vías centrales al año ${ }^{2}$.

Si bien se ha demostrado la superioridad de la punción yugular guiada por ultrasonido, la canulación guiada por reparos anatómicos es muy frecuente y presenta, en manos experimentadas, una alta tasa de éxito técnico (hasta el 99\% de éxito reportado)3. Sin embargo, la co- locación de un catéter venoso central conlleva el riesgo de lesionar estructuras adyacentes como pleura, esófago, nervios o arterias adyacentes.

La punción arterial accidental con aguja ocurre en cerca del 5\% de las canulaciones venosas y usualmente no ocasiona secuelas clínicas. Sin embargo, la colocación inadvertida de un catéter de grueso calibre en el sistema arterial del cuello es menos frecuente (incidencia de 0,1 a $0,8 \%$ ) pero puede llevar a complicaciones graves. Dentro de estas podemos incluir hematomas (que pueden expandirse y comprometer la vía aérea), hemotórax, pseudoaneurismas, fístulas AV y stroke ${ }^{4,5}$.

Cuando se advierte la colocación accidental de un catéter en arteria, este debe ser removido inmediatamente debido al riesgo de formación de trombos con la canulación prolongada ${ }^{6}$. Para ello, la recomendación actual es el manejo quirúrgico o endovascular, ya que la compresión manual se asocia a una alta tasa de complicaciones graves 6 .

Sin embargo, cuando la injuria arterial ha pasado inadvertida, como en el presente caso, la extracción del catéter precipita una situación catastrófica. Dependiendo del grado de inestabilidad del paciente y de la localización del trauma vascular puede optarse por una estrategia quirúrgica o endovascular. Pacientes con severo compromiso hemodinámico y respiratorio como el presentado en este artículo y aquellos con lesiones por detrás o debajo de la clavícula se benefician con el manejo endovascular por sobre el quirúrgico.

Para la resolución de emergencia del presente caso seleccionamos un stent de acero inoxidable cubierto de PTFE expandible por balón (ADVANTA V12. Atrium Interventional Surgery, Hudson, New Hampshire), 9-59 mm con el que se logró la resolución del sangrado activo y la rápida estabilización clínica del paciente.

\section{CONCLUSIONES}

El tratamiento endovascular con stents grafts de las lesiones vasculares iatrogénicas del cuello debe ser considerado de primera línea para el manejo del paciente crítico o con localizaciones de difícil acceso para la cirugía.

\section{BIBLIOGRAFÍA}

1. GiswoldME, et al. latrogenic arterial injury is an increasingly important cause of arterial trauma. The American Journal of Surgery Volume 187, Issue 5, 590-93.

2. OncuS, Ozsut H, Yildirim A, Ay P, Cakar N, Eraksoy H, et al. Central venous catheter related infections: risk factors and the effect of glycopeptide antibiotics. Ann Clin Microbiol Antimicrob 2003;2:3.

3. OliverWC Jr, Nuttall GA, Beynen FM, Raimundo HS, Abenstein JP, ArnoldJJ. The incidence of artery puncture with central venous cannulation using a modified technique for detection and prevention of arterial cannulation. J Cardiothorac Vasc Anesth 1997;11:851-5.

4. Golden LR. Incidence and management of large-bore introducer sheath puncture of the carotid artery. I Cardiothorac Vasc Anesth 1995;9: 425-8

5. Reuber M, Dunkley LA, Turton EP, Bell MD, Bamford JM. Stroke after internal jugular venous cannulation. Acta Neurol Scand 2002;105: 235-9.

6. Guilbert M-C, et al. Arterial trauma during central venous catheter insertion: Case series, review and proposed algorithm. Journal of Vascular Surgery Volume 48, Issue 4, 918 - 925. 